\title{
Preoperative Pain Management of Patients with Hip Fractures: Blind Fascia Iliaca Compartment Block Compared to Ultrasound Guided Femoral Nerve Block-A Randomized Controlled Trial
}

\author{
Johanne Bangshoej*, Thomas Thougaard, Hans Fjeldsøe-Nielsen, Sandra Viggers \\ Department of Anesthesiology, Nykoebing Falster Hospital, Nykoebing Falster, Denmark \\ Email: ${ }^{*}$ johanne.bangshoej@gmail.com
}

How to cite this paper: Bangshoej, J., Thougaard, T., Fjeldsøe-Nielsen, H. and Viggers, S. (2020) Preoperative Pain Management of Patients with Hip Fractures: Blind Fascia Iliaca Compartment Block Compared to Ultrasound Guided Femoral Nerve Block-A Randomized Controlled Trial. Open Journal of Anesthesiology, 10, 371-380.

https://doi.org/10.4236/ojanes.2020.1011033

Received: September 19, 2020

Accepted: November 7, 2020

Published: November 10, 2020

Copyright $\odot 2020$ by author(s) and Scientific Research Publishing Inc. This work is licensed under the Creative Commons Attribution International License (CC BY 4.0).

http://creativecommons.org/licenses/by/4.0/ (c) (i) Open Access

\begin{abstract}
Introduction: Blind fascia iliaca compartment block (FICB) and ultrasound guided femoral nerve block (FNB) are two types of peripheral nerve blocks, commonly used in preoperative pain management in patients with hip fractures in Danish emergency departments. The aim of this study was to compare the efficacy in pain management of these two types of peripheral nerve blocks in the preoperative period in patients with hip fractures. Method: We performed a randomized controlled study. The primary outcome was the proportion of patients with a numeric rating scale (NRS) pain score equal to three or less at rest and after passive leg raise test three hours after block administration. Results: A total of 88 patients were included in the study and 67 patients in the statistical analysis with 33 in the FICB group and 34 in the FNB group. The results showed a significant reduction in the proportion of patients with an NRS score higher than three, three hours after administration of either FICB or FNB compared to at inclusion. There was no significant difference in pain scores between patients receiving FICB versus patients receiving FNB at rest or after passive leg raise $(\mathrm{p}=0.25$ and $\mathrm{p}=0.86$, respectively). Conclusion: Blind FICB and ultrasound guided FNB were effective in preoperative pain management in patients with hip fractures. The results showed that the two types of peripheral nerve blocks were equally efficient in providing pain management in the preoperative period.
\end{abstract}

\section{Keywords}

Local Analgesia, Peripheral Nerve Block, Pain Control, Preoperative Optimization 


\section{Introduction}

Hip fracture is a common injury in the geriatric population, resulting in approximately 10,000 admissions per year in Denmark [1]. Patients with hip fractures often have a high comorbidity burden, including diabetes, renal dysfunction and dementia, and the mortality increases from $10 \%$ at one month to $30 \%$ after one year [2] [3] [4]. The high comorbidity burden and advanced age of the population increase the risk of general anesthesia in this population [3] [5].

Hip fractures are painful and sufficient preoperative pain management remains a challenge. Peripheral nerve blocks have been shown to be effective in reducing preoperative pain and decreasing systemic side effects compared to conventional oral and parenteral analgesia such as non-steroidal anti-inflammatory drugs (NSAIDs) and opioids [4] [6] [7].

Studies have shown that regional anesthesia, including neuraxial and peripheral nerve blocks, is associated with lower incidence of pulmonary complications, postoperative cognitive dysfunction and a shorter length of stay in the intensive care unit (ICU) in this population [4] [8] [9].

Blind fascia iliaca compartment block (FICB) and ultrasound guided femoral nerve block (FNB) are two types of peripheral nerve blocks commonly used in preoperative pain management in Danish emergency departments (EDs). Both techniques anesthetize the nerves responsible for innervating the hip by using either a landmarked based or ultrasound guided technique, respectively [7] [10]. Potential complications of peripheral nerve blocks are nerve- and vascular damage and systemic toxicity to local anesthetics [9]. The theoretical advantage of using an ultrasound guided technique is that visualizing anatomic structures lowers the risk of nerve damage and vascular perforation [11]. However, the incidence of reported complications and adverse events is low for both techniques [10] [12].

Uncertainty remains regarding the potential superiority of one peripheral nerve block compared to the other. Accordingly, the primary aim of this study was to compare the efficacy in pain management of the blind FICB versus the ultrasound guided FNB in patients with hip fractures in the preoperative period. We hypothesized that patients with hip fractures receiving ultrasound guided FNB would report lower pain scores compared to blind FICB.

\section{Methods}

This randomized controlled trial was conducted from April 2018 to May 2019 in the ED of Nykoebing Falster Hospital, Region Zealand, Denmark. The study population included patients 18 years of age or older with a suspected hip fracture. Exclusion criteria included allergy to the local analgesic drug, infection at the intended injection site, an international normalized ratio (INR) greater than two, daily treatment with non-vitamin $\mathrm{K}$ oral anticoagulants (NOACs), multi trauma patients with more than two fractures, and patients suffering from dementia or lack of ability to perform a pain score using the numeric rating scale 
(NRS). The study was not blinded.

Patients were randomized to either blind FICB or ultrasound guided FNB with respectively 40 milliliters $(\mathrm{ml})$ and $15 \mathrm{ml}$ of Marcaine $0.25 \%$. Marcaine was used because it was the drug of choice for the standard blind FICB in the ED. For pragmatic reasons, we used a fixed volume in each group instead of adjusting according to individual bodyweight. By using ultrasound, a smaller volume of Marcaine was used in the FNB group compared to the FICB group. Both procedures were performed by anesthetists who were trained in performance of peripheral nerve blocks with experience ranging from intermediate to experts. FICB was performed using a needle insertion two centimeters below the point between the lateral third and medial two thirds of a line between the pubic tubercle and anterior superior iliac spine. Two succeeding loss of resistance 'pops' were elicited as the needle punctured the fascia lata and fascia iliaca. To perform the FNB, the femoral nerve was identified by ultrasound and Marcaine was injected under continuous ultrasound in plane needle visualisation. Aspiration checks were performed for every five $\mathrm{ml}$ of Marcaine injected in both blocks.

The peripheral nerve block was performed prior to $\mathrm{x}$-ray and eventual epidural catheter insertion in order to reduce pain associated with mobilization to these two procedures. If the x-ray confirmed the suspicion of a hip fracture, the patient was transferred either via the post anesthetic care unit (PACU) for preoperative epidural catheter insertion or directly to the orthopedic ward if an epidural catheter was contraindicated. If a hip fracture was not confirmed on x-ray, the patient was excluded from the study.

The NRS pain scores were recorded immediately before the intervention and again three hours later. The NRS is a subjective pain scale score, where zero refers to no pain and 10 to extreme pain [13]. In the literature, a peripheral nerve block is usually considered sufficient if the NRS score is equal to three or lower [1] [11]. The pain score was measured at rest and after a passive leg raise test, where the fractured limb was passively elevated to 15 degrees from supine position. We used this dynamic pain score because not all patients were suspected to be in pain at rest [12]. The orthopedic surgeon was responsible for administering additional analgesia after local guidelines based on the NRS.

The primary outcome was the proportion of patients with an NRS pain score equal to three or lower, three hours after intervention at rest and after passive leg raise. The secondary outcomes were opioid administration after the intervention and until the next pain score, adverse events to the peripheral nerve block and patient discomfort on a five-point verbal rating scale (VRS) with zero representing no discomfort and four very severe discomfort.

The study was approved by the regional ethics committee (reference number SJ-655) and by the regional data monitoring committee (reference number REG-190-2017) in Denmark. Written and verbal informed consent was obtained from all patients.

\section{Statistical analysis plan}

The trial was powered towards the binary primary endpoint NRS pain score 
equal to three or lower three hours after intervention. Assuming that $70 \%$ of patients with ultrasound guided FNB would achieve an NRS pain score equal to three or lower and that $40 \%$ of patients with blind FICB would achieve an NRS pain score equal to three or lower, the trial would achieve a power of $80 \%$ with an alpha-level of 0.05 with the inclusion of 80 patients. To adjust for drop-outs and missingness 88 patients were included.

Randomization was done with an internet-based program randomization.com [14] with one to one fashion to either blind FICB or ultrasound guided FNB, with 44 patients allocated to each group.

All analyses were conducted on the per protocol population. Categorical variables were presented as frequencies, and continuous variables as means with standard deviation (SD). Differences in NRS score equal to three or lower, from inclusion and until three hours after administration of block within treatment allocations were analyzed by the McNemar paired test. The primary outcome was compared using the Chi-Squared test. Data were analyzed using IBM SAS Enterprise Guide version 7.1.

\section{Results}

A total of 88 patients were included in the study. The Danish Personal Identification Number was not registered for 12 patients. A total of nine patients had missing pain scores leaving 67 (76\%) patients for inclusion in the analysis with 33 in the FICB group and 34 in the FNB group. A flowchart displaying inclusions is shown in Figure 1. No patients were excluded because of missing fracture. Baseline characteristics were balanced between groups and are shown in Table 1. The incidence of hip fracture was greater among women than men.

The distribution of patient-reported NRS scores after inclusion and after three hours can be seen in Figure 2. After inclusion, 20 patients (61\%) randomized to FICB and 17 patients (50\%) to FNB had an NRS pain score greater than three at rest. Three hours after receiving the block according to randomization, $12 \mathrm{pa}-$ tients (36\%) receiving FICB had an NRS pain score greater than three at rest ( $\mathrm{p}$ $=0.01)$ compared to $8(24 \%)$ in the FNB group $(\mathrm{p}=0.003)$.

After inclusion, all 67 patients (100\%) had an NRS score greater than three after

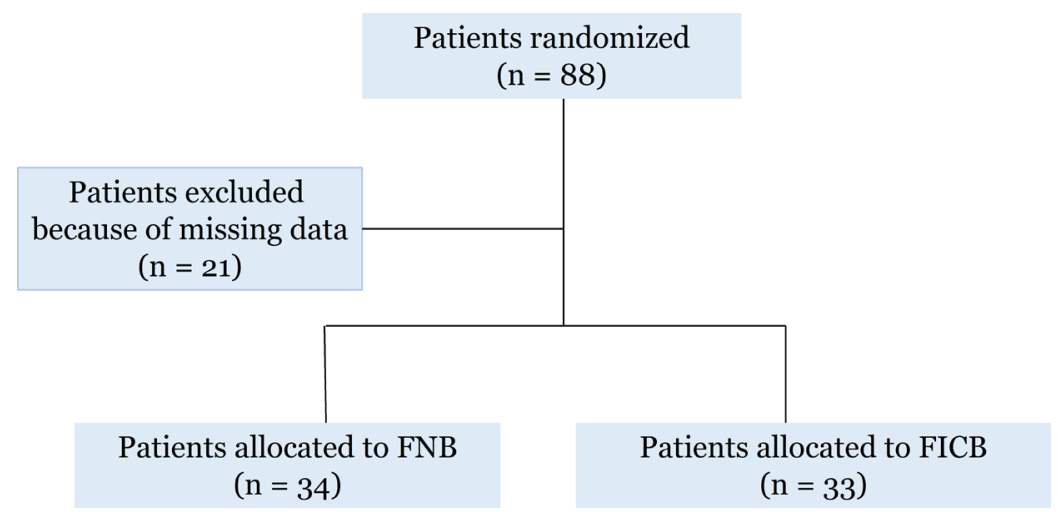

Figure 1. Patients included. 
Table 1. Demographics.

\begin{tabular}{ccc}
\hline & $\begin{array}{c}\text { Fascia iliaca compartment } \\
\text { block }(\mathbf{n}=33)\end{array}$ & $\begin{array}{c}\text { Femoral nerve } \\
\text { block }(\mathbf{n}=34)\end{array}$ \\
\hline Sex, $\mathbf{n}(\%)$ & & \\
Male & $9(27 \%)$ & $10(29 \%)$ \\
Female & $24(73 \%)$ & $24(71 \%)$ \\
Age in years, mean (SD) & $76 \pm 12$ & $76 \pm 10$ \\
Time for operation in hours, mean (SD) & $14 \pm 8.4(\mathrm{n}=32$, one missing $)$ & $16 \pm 5.7$ \\
\hline
\end{tabular}
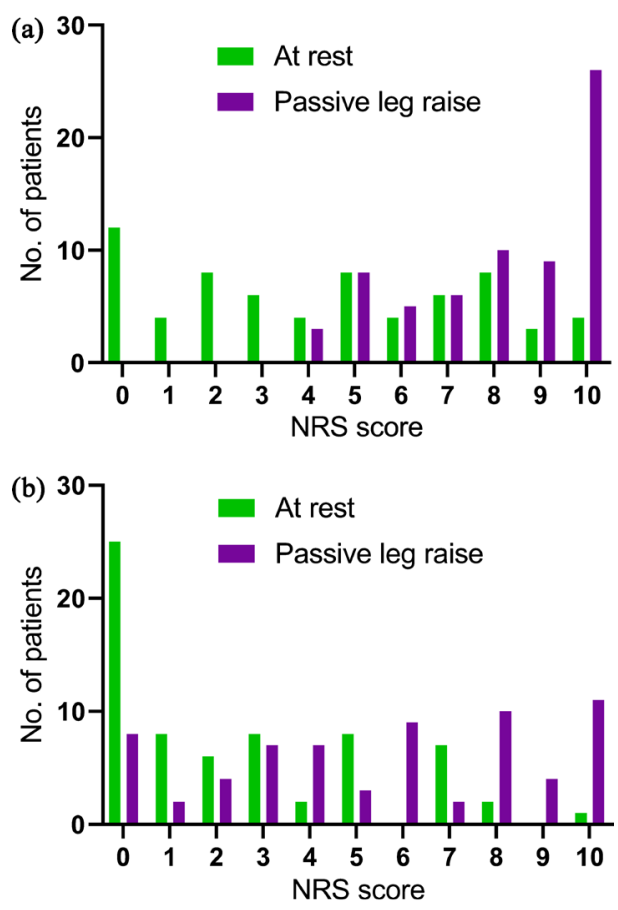

Figure 2. Distribution of patient reported NRS scores at rest and after passive leg raise upon randomization (a) and after three hours (b).

passive leg raise. Three hours after receiving the block according to randomization, 23 patients (70\%) receiving FICB had an NRS pain score greater than three after passive leg raise compared to $23(68 \%)$ patients in the FNB group. The proportion of patients with an NRS above or equal to three are shown in Figure 3. Only one patient in the FICB group was reported to receive additional opioids (15 mg Oxynorm), during the first three hours after the intervention. Only one patient in the FNB group reported discomfort of one on the VRS, during the procedure. No adverse effects were reported.

\section{Discussion}

This study showed a significant pain reduction in patients with an initial NRS pain score greater than three at inclusion compared to three hours after the administration of either blind FICB or ultrasound guided FNB, both at rest and after passive leg raise. Some patients with hip fractures did not feel pain at rest but 


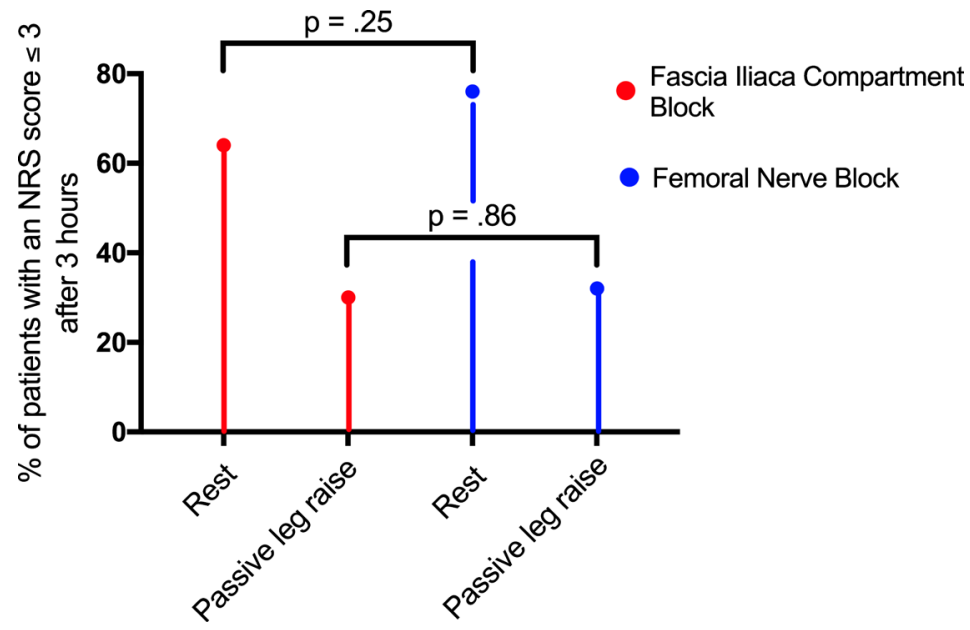

Figure 3. Primary outcome. Percent of patients with an NRS score below or equal to three after three hours.

had an NRS score greater than three after passive leg raise before block administration. It is worth noting that before block administration at rest, the number of patients having pain was higher in the FICB group compared to the FNB group. Three hours after the intervention there was no significant difference in the proportion of patients with an NRS pain score equal to three or lower between the two groups, both at rest and after passive leg raise. All included patients received a peripheral nerve block after arrival to the ED and were operated within 15 hours, which is in accordance with national guidelines.

Prior studies have compared the efficacy of the two types of peripheral nerve blocks when performed as a continuous block and postoperatively [2] [15] [16]. Only two studies have compared different peripheral nerve blocks administered as a single shot in the preoperative period [17] [18]. In these two studies the maximum time point for pain score was two hours after intervention and the FNB was either performed with an ultrasound or a nerve stimulator guided technique. In our study the pain score was recorded after three hours which seems more representative of the preoperative period and we only used an ultrasound guided technique to perform the FNB because a nerve stimulator is less frequently used at our institution and would cause painful twitching of the quadriceps muscle in the fractured limb [7] [19].

In most studies, the primary outcome is pain score measured by NRS or the visual analogue scale (VAS). By using subjective pain score scales many studies exclude participants with cognitive impairment and dementia because patients are not able to understand or express their pain score [3]. Excluding this patient group makes the study result less representative, however, the strength of using the subjective pain score scales is that it is a simple and reproducible method [13]. In one study, the authors claim that VAS is validated for use in patients with impaired cognition while other parameters such as body expression, vital parameters and opioid consumption are advantageous, especially in people with severe dementia [3]. In the present study, we chose to use NRS because the scale 
is both visual and verbal and therefore better suitable for the geriatric patient population with possible cognitive impairment compared to VAS which is only visual [13].

A previous study demonstrated superior efficacy of the nerve stimulated guided FNB, however, the authors conclude that the blind FICB was cheaper and quicker to teach, perform and learn [17].

In our study, the percentage of successful blocks is low compared to previous studies (81\% - 96\%) [12]. Another study also reported a low success rate comparable to the rate in our study (67\%) [12]. One of their explanations was that the blocks were performed by an anesthetist in training. The same could potentially explain the low success rate in our study because the blocks were performed by 19 different anesthetists with different experience in nerve block performance. However, they all had prior training by an expert with many years of experience. Marcaine is used in over $50 \%$ of the studies comparing the two types of peripheral nerve blocks, which exclude the drug as being the reason for the low success rate [2] [3] [7] [10] [11] [17] [18] [20].

In our study, the patients were transferred to the ICU if the x-ray showed a hip fracture and the patient had no contraindications to epidural analgesia. An advantage of the peripheral nerve block is that it can improve mobilization and positioning and therefore lead to a better quality of $\mathrm{x}$-ray image and successful epidural catheter insertion [4] [19]. If the epidural was placed before measuring the NRS pain score three hours after block administration, the success rate of the peripheral nerve block may have been higher because the epidural catheter provides effective analgesia in both limbs. Only four patients in each of the two study groups had an epidural catheter inserted before measuring NRS pain score after three hours. Furthermore, only two patients in the FNB group and one patient in the FICB group had surgery within the first three hours so these factors probably have no influences on the low success rate.

A limitation of this study was the high proportion of missing data. Of the recruited patients only $76 \%$ were included for data analysis. This could be attributed to organizational and logistic reasons in the hospital and between the respective departments. In our study, the on-call anesthetist was supposed to include the patients and collect data. As the hospital only had one anesthetist on call, the data could not be collected if the anesthetist was called for another more urgent emergency situation. The proportion of patients with hip fracture and dementia is high, about $30 \%-45 \%$ which is another limitation in this study as patients with dementia were not included due to ethical considerations [3]. Finally, the lack of blinding could potentially bias the results, however, since the primary outcome was patient-reported pain scores, and since both allocation groups underwent a procedure (i.e. no placebo group), we consider this less likely.

\section{Conclusions}

Since there was no significant difference between the two blocks in our study, we 
do not find the blind FICB preferable because most EDs have ultrasound machines available which can visualize nerves and vascular structures and therefore minimize potential damage. Another advantage of the ultrasound visualization is that it permits a smaller volume of local analgesic drugs to be used but remains efficacy and reduces potential adverse effects of the larger volume of local analgesic drugs used in the blind FICB.

Initially, we hypothesized that patients with hip fractures receiving ultrasound guided FNB would report lower pain score compared with blind FICB. In summary, the study showed that either a blind FICB or ultrasound guided FNB is equally useful in suspected hip fractures in the ED. There were no adverse events and only one patient reported slight discomfort to the procedure.

\section{Acknowledgements}

We are grateful to Tobias Lyngeraa, $\mathrm{MD}, \mathrm{PhD}$, for providing assistance with the study protocol and also to the staff that recruited, included and randomised patients, and subsequently collected the data for the study. We would like to thank Sebastian Wiberg, MD, $\mathrm{PhD}$, for his contributions to the statistical analyses.

\section{Conflicts of Interest}

The authors declare no conflicts of interest regarding the publication of this paper.

\section{References}

[1] Hauritz, R.W., Gerlif, C. and Ronholm, E. (2009) Fascia Iliaca Block Performed by Emergency Department Physician Trainees in Hip Fractures. Ugeskrift for Læger, 171, 515-518.

[2] Sahota, O., Rowlands, M., Bradley, J., Van de Walt, G., Bedforth, N., Armstrong, S., et al. (2014) Femoral Nerve Block Intervention in Neck of Femur Fracture (FINOF): Study Protocol for a Randomized Controlled Trial. Trials, 15, Article No. 189. https://doi.org/10.1186/1745-6215-15-189

[3] Unneby, A., Svensson, O., Gustafson, Y. and Olofsson, B. (2017) Femoral Nerve Block in a Representative Sample of Elderly People with Hip Fracture: A Randomised Controlled Trial. Injury, 48, 1542-1549. https://doi.org/10.1016/j.injury.2017.04.043

[4] Madabushi, R., Rajappa, G.C., Thammanna, P.P. and Iyer, S.S. (2016) Fascia Iliaca Block vs Intravenous Fentanyl as an Analgesic Technique before Positioning for Spinal Anesthesia in Patients Undergoing Surgery for Femur Fractures-A Randomized Trial. Journal of Clinical Anesthesia, 35, 398-403. https://doi.org/10.1016/j.jclinane.2016.09.014

[5] Neuman, M.D., Silber, J.H., Elkassabany, N.M., Ludwig, J.M. and Fleisher, L.A. (2012) Comparative Effectiveness of Regional versus General Anesthesia for Hip Fracture Surgery in Adults. Anesthesiology, 117, 72-92. https://doi.org/10.1097/ALN.0b013e3182545e7c

[6] Fujihara, Y., Fukunishi, S., Nishio, S., Miura, J., Koyanagi, S. and Yoshiya, S. (2013) Fascia Iliaca Compartment Block: Its Efficacy in Pain Control for Patients with Proximal Femoral Fracture. Journal of Orthopaedic Science, 18, 793-797. 
https://doi.org/10.1007/s00776-013-0417-y

[7] Beaudoin, F.L., Haran, J.P. and Liebmann, O.A (2013) Comparison of Ultrasound-Guided Three-in-One Femoral Nerve Block versus Parenteral Opioids Alone for Analgesia in Emergency Department Patients with Hip Fractures: A Randomized Controlled Trial. Academic Emergency Medicine, 20, 584-591.

[8] Zywiel, M.G., Prabhu, A., Perruccio, A.V. and Gandhi, R. (2014) The Influence of Anesthesia and Pain Management on Cognitive Dysfunction after Joint Arthroplasty: A Systematic Review. In: Leopold, L.S., Ed., Clinical Orthopaedics and Related Research, Springer, Berlin, 1453-1466. https://doi.org/10.1007/s11999-013-3363-2

[9] Yun, M.J., Kim, Y.H., Han, M.K., Kim, J.H., Hwang, J.W. and Do, S.H. (2009) Analgesia before a Spinal Block for Femoral Neck Fracture: Fascia Iliaca Compartment Block. Acta Anaesthesiologica Scandinavica, 53, 1282-1287.

[10] Lees, D., Harrison, W.D., Anker,s T., A'Court, J., Marriott, A., Shipsey, D., et al. (2016) Fascia Iliaca Compartment Block for Hip Fractures: Experience of Integrating a New Protocol across Two Hospital Sites. European Journal of Emergency Medicine, 23, 12-18.

[11] Lee, H.K., Kang, B.S., Kim, C.S. and Choi, H.J. (2014) Ultrasound-Guided Regional Anesthesia for the Pain Management of Elderly Patients with Hip Fractures in the Emergency Department. Clinical and Experimental Emergency Medicine, 1, 49-55. https://doi.org/10.15441/ceem.14.008

[12] Foss, N.B., Kristensen, B.B., Bundgaard, M., Bak, M., Heiring, C., Virkelyst, C., et al. (2007) Fascia Iliaca Compartment Blockade for Acute Pain Control in Hip Fracture Patients: A Randomized, Placebo-Controlled Trial. Anesthesiology, 106, 773-778. https://doi.org/10.1097/01.anes.0000264764.56544.d2

[13] Hawker, G.A., Mian, S., Kendzerska, T. and French, M. (2011) Measures of adult pain: Visual Analog Scale for Pain (VAS Pain), Numeric Rating Scale for Pain (NRS Pain), McGill Pain Questionnaire (MPQ), Short-Form McGill Pain Questionnaire (SF-MPQ), Chronic Pain Grade Scale (CPGS), Short Form-36 Bodily Pain Scale (SF-36 BPS), and Measure of Intermittent and Constant Osteoarthritis Pain (ICOAP). Arthritis Care \& Research, 63, 240-252.

[14] Randomization.com (2017) Randomization Generators and Plans. http://randomization.com

[15] Capdevila, X., Biboulet, P., Bouregba, M., Barthelet, Y., Rubenovitch, J. and d'Athis, F. (1998) Comparison of the Three-in-One and Fascia Iliaca Compartment Blocks in Adults: Clinical and Radiographic Analysis. Anesthesia \& Analgesia, 86, 1039-1044.

[16] Yu, B., He, M., Cai, G.Y., Zou, T.X. and Zhang, N. (2016) Ultrasound-Guided Continuous Femoral Nerve Block vs Continuous Fascia Iliaca Compartment Block for Hip Replacement in the Elderly: A Randomized Controlled Clinical Trial (CONSORT). Medicine (Baltimore), 95, e5056.

[17] Newman, B., McCarthy, L., Thomas, P.W., May, P., Layzell, M. and Horn, K.A (2013) Comparison of Pre-Operative Nerve Stimulator-Guided Femoral Nerve Block and Fascia Iliaca Compartment Block in Patients with a Femoral Neck Fracture. Anaesthesia, 68, 899-903.

[18] Reavley, P., Montgomery, A.A., Smith, J.E., Binks, S., Edwards, J., Elder, G., et al. (2015) Randomised Trial of the Fascia Iliaca Block versus the "3-in-1" Block for Femoral Neck Fractures in the Emergency Department. Emergency Medicine Journal, 32, 685-689. https://doi.org/10.1136/emermed-2013-203407

[19] Ranjit, S. and Pradhan, B.B. (2016) Ultrasound Guided Femoral Nerve Block to Provide Analgesia for Positioning Patients with Femur Fracture before Subarach- 
noid Block: Comparison with Intravenous Fentanyl. Kathmandu University Medical Journal, 14, 125-129.

[20] Williams, H., Paringe, V., Shenoy, S., Michaels, P. and Ramesh, B. (2016) Standard Preoperative Analgesia with or without Fascia Iliaca Compartment Block for Femoral Neck Fractures. Journal of orthopaedic surgery (Hong Kong), 24, 31-35.

https://doi.org/10.1177/230949901602400109 